\title{
Epispadias Repair after Failed Surgery in Childhood
}

\author{
Miroslav Djordjevic ${ }^{1}$ Vladimir Kojovic ${ }^{1}$ Marta Bizic $^{1} \quad$ Marko Majstorovic $^{1}$ Vojkan Vukadinovic $^{1}$ \\ Gradimir Korac $^{1}$ Zoran Krstic ${ }^{1}$ \\ ${ }^{1}$ School of Medicine, University of Belgrade, Belgrade, Serbia \\ Address for correspondence and reprint requests Marta Bizic, MD, \\ School of Medicine, University of Belgrade, Tirsova 10, \\ Eur J Pediatr Surg 2013;23:67-71. \\ Belgrade 11000, Serbia (e-mail: martabizic@uromiros.com).
}

\begin{abstract}
Keywords

- epispadias

- redo surgery

- penile disassembly

Introduction Redo surgery in failed epispadias presents a great challenge. Our aim was to present a radical approach for correction of penile deformities as well as urethral reconstruction in patients after failed epispadias repair.

Materials and Methods Between January 2006 and January 2011, 13 patients, aged 13 to 22 years, underwent redo surgery due to failed epispadias repair in childhood. All patients presented with severe dorsal curvature and short urethra. First stage included penile disassembly technique with complete separation of corporal bodies, urethral dissection, and transposition and subtotal glans mobilization. Residual dorsal curvature was corrected by tunical incision and grafting of the defect. Short urethra was dissected and transposed ventrally with opening at the base of the penis. Penile entities were reassembled in normal anatomical relationship. Penile body was covered using available vascularized skin flaps. After 6 months, second stage was performed and included reconstruction of the penile urethra using buccal mucosa graft and scrotal hairless skin flap.

Results Follow-up ranged from 12 to 60 months (mean 33 months). Acceptable outcome is achieved in all the patients. Complete penile lengthening and straightening is obtained in 10 out of 13 patients. Mild curvature is noted in three patients without consequences. Satisfactory sexual activity was reported from nine patients. One patient developed fistula that was closed after 4 months, whereas all other patients reported normal voiding with no difficulties.

Conclusions Redo surgery of failed epispadias is very demanding procedure. Radical approach in these cases is necessary for complete repair of all penile deformities with satisfactory postoperative outcome.
\end{abstract}

\section{Introduction}

Epispadias presents the most severe congenital anomaly of the penis that requires advanced surgical skills for successful repair and satisfactory outcome. The main hallmark of the epispadias is partially or completely opened urethra on the dorsal side of the penis, usually combined with marked dorsal curvature. Glans is always opened dorsally and complete penile body is significantly shortened. ${ }^{1}$ The goal of epispadias repair is reconstruction of functionally and cosmetically acceptable penis while attaining the maximum corporal length as possible. Despite constant improvement in surgical approach, high incidence of failure rate is widely present due to rarity of this anomaly and severity of penile deformities. Usually, patients undergo several surgical procedures in received

May 18, 2012

accepted after revision

August 30, 2012

published online

November 19, 2012 
childhood with questionable results. Outcome can be severely worsened after penile growth in puberty due to increasing of residual dorsal curvature. Inadequate penile length becomes more obvious in adolescents and their expectations for functional and aesthetically acceptable penis impose successful redo epispadias surgery. Our aim was to present a radical approach for correction of all penile deformities in patients after epispadias repair in childhood.

\section{Patients/Materials and Methods}

Between January 2006 and January 2011, 13 patients, aged 13 to 22 years, underwent redo surgery due to failed epispadias repair in childhood. The number of previous repairs in childhood ranged from one to five (median 2.2). All patients had severe dorsal curvature and short penile urethra and were continent before redo surgery ( - Fig. 1). Mean preoperative penile length was $9.2 \pm 1.1 \mathrm{~cm}$ (ranged 6.9 to $10.3 \mathrm{~cm}$ ). Patients were operated in two-stage procedure. First stage included penile disassembly and grafting procedure for complete straightening and lengthening of the penis, and second stage included reconstruction of the penile urethra using buccal mucosa graft and scrotal hairless skin flap.

\section{Operative Technique}

Standard circumferential incision was made under the glans. Complete penile degloving was performed carefully to avoid injury of the skin. All scars around corporal bodies, from the previous surgeries, were released. After degloving, severely deformed corporal bodies were identified, and the urethra was very short and positioned dorsally. Lifting of the neurovascular bundle began on the ventrolateral side of the penis. After lifting the neurovascular bundle, subtotal glans mobilization was performed for additional release of corporal bodies. Subcoronal plexus of the glans was preserved by meticulous dissection. Separation of the corporal bodies was done along with the dissection of the urethra. In this way, penile disassembly was completed. Artificial erection or erection induced by prostaglandin E1 injection revealed all deformities of the corporal bodies. Short ure-

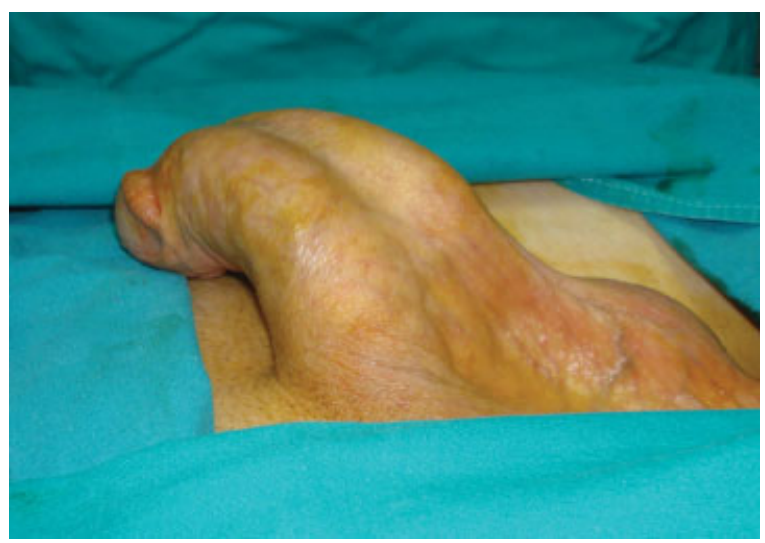

Fig. 1 Appearance of the treated epispadias in childhood. Severe curvature is visible in erect penis.

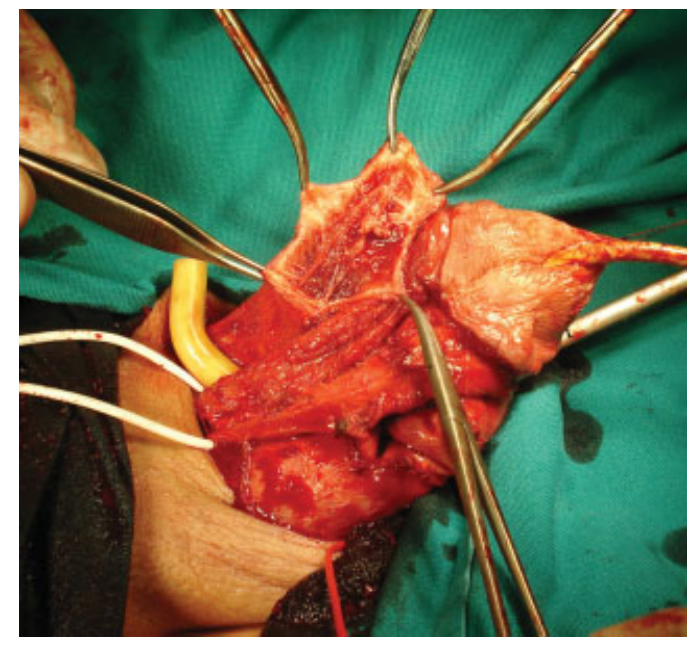

Fig. 2 Penile disassembly was done. Corpora cavernosa were lengthened and straightened by penile tunical incision and grafting.

thra, which was limiting factor for straightening of the penis, was transected at subcoronal level. Complete straightening of the corporal bodies was achieved by incision made on the dorsal surface of the tunica albuginea, directly opposite to the site of maximal curvature. The defects of the tunica albuginea were closed by bovine or equine pericardium (-Fig. 2). Erection was re-established, and good result of penile curvature correction was confirmed. Corporal bodies were sutured together and neurovascular bundle was fixed on its anatomical position. Urethral remnant was transposed ventrally and urethral orifice was fixed at the base of the penis (-Fig. 3). Gap between glanular part of the urethra and urethral stump was left for the second stage repair. Penile entities were

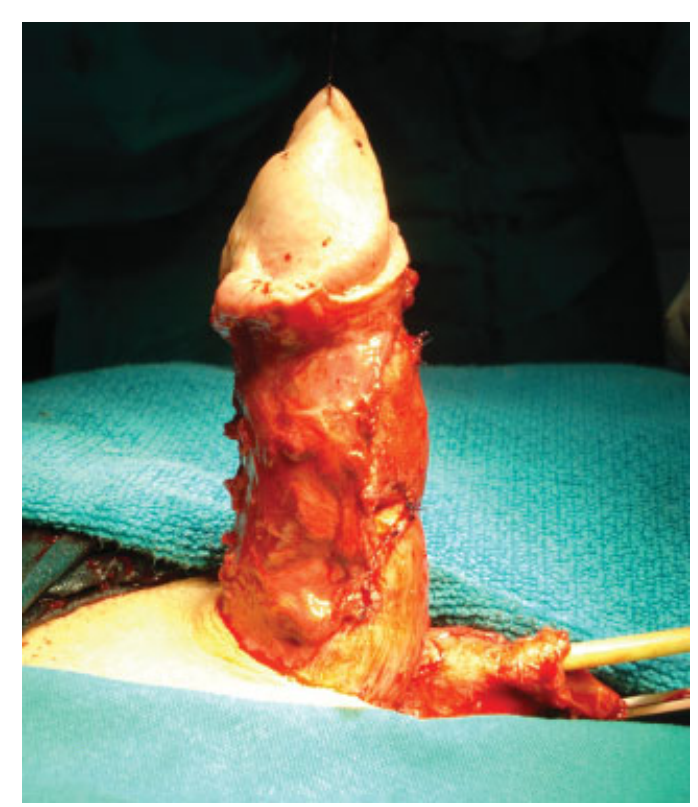

Fig. 3 Corpora cavernosa are joined, neurovascular bundles are placed dorsally, and glans is fixed in proper position. Short urethra is repositioned ventrally at the penoscrotal angle. 


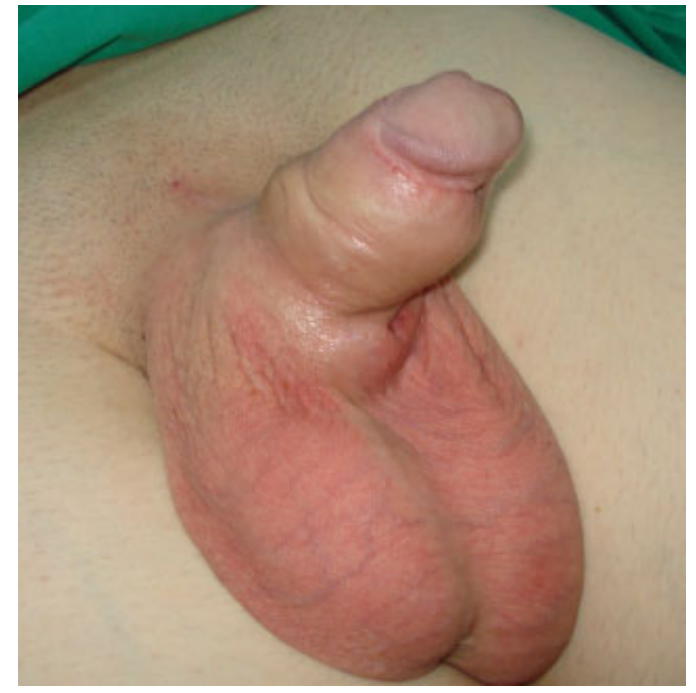

Fig. 4 Appearance 6 months after surgery. Hypospadiac urethra is visible at the base of the penis.

reassembled in normal anatomical relationship. Penile skin was reconstructed using remaining skin flaps. At the end of the first stage, complete straightening and lengthening of the penis was achieved while urethral orifice was positioned at the base of the penis on its ventral side. After 6 months, patients underwent second stage procedure that included repair of resultant hypospadias (-Fig. 4). Longitudinal scrotal island skin flap with abundant vascular pedicle was carefully harvested. If needed, patient underwent laser epilation of the scrotal skin before surgery. Buccal mucosal graft was taken from the inner patient's cheek and fixed to the corporal bodies to create dorsal part of the newly reconstructed urethra. Previously harvested longitudinal island scrotal skin flap was joined with mucosal graft over 16 -Fr catheter using $5 / 0$ poliglecaprone 25 running suture and anastomosed to glanular part of urethra ( - Fig. 5). In this way, missing urethra was formed. A very wide and abundant pedicle flap covered all suture lines to prevent fistula formation. Remaining penile and scrotal skin was used to cover penile shaft. Finally, penis of satisfactory size and shape was created together with complete reconstruction of the urethra ( - Fig. 6). Urethral stent was left for 2 weeks, while urinary drainage was enabled by suprapubic tube.

\section{Results}

Mean follow-up was 33 months (ranged from 12 to 60 months). There was no injury of the neurovascular bundles and no sign of corporal or glans necrosis. Patients were followed by treating surgeon at $1,3,6$, and 12 months and yearly thereafter. Follow-up included measurement of the penile length as well as voiding function. Last appointment included a questionnaire about surgery outcome. Ten patients have completely straightened and lengthened penis. Three patients manifested mild curvature that did not require

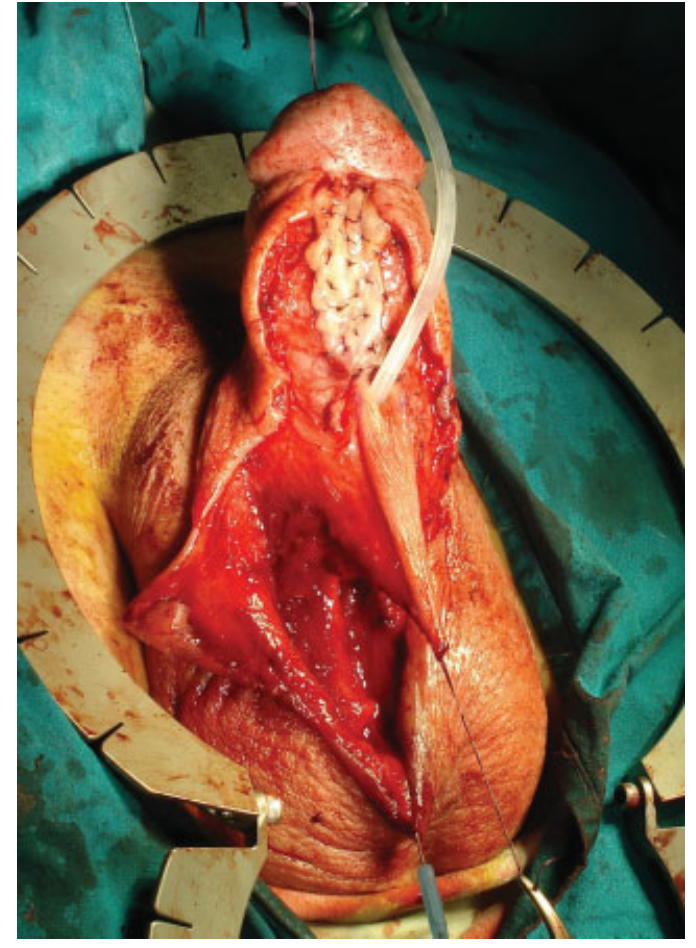

Fig. 5 Urethral lengthening is done by combined buccal mucosa graft and longitudinal scrotal skin flap.

further treatment. Nine patients were sexually active and they reported satisfactory sexual intercourse. One patient developed fistula that was closed 4 months later, while other patients reported regular voiding with no difficulties (-Table 1).

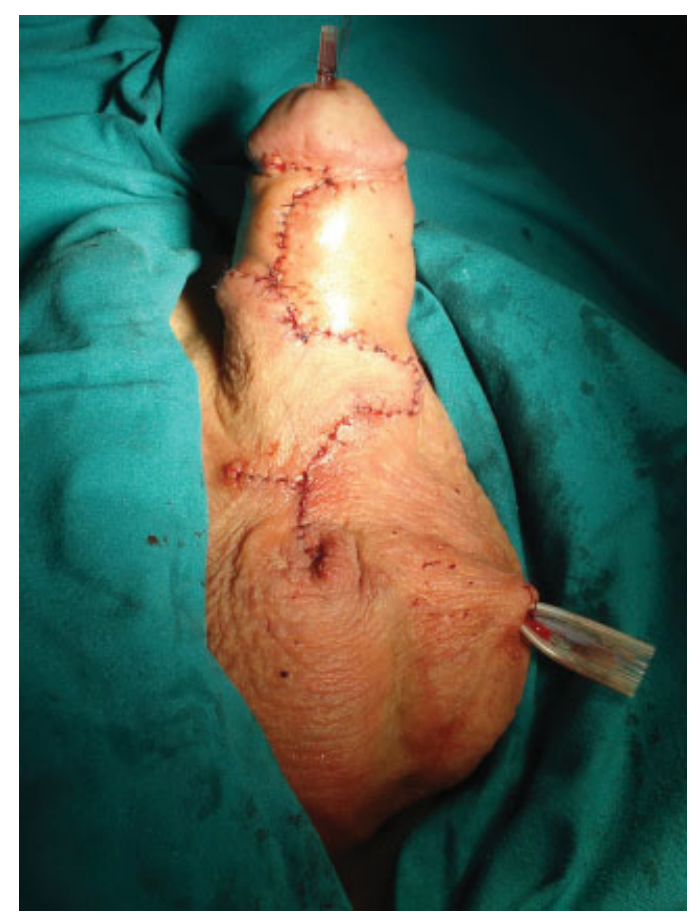

Fig. 6 Appearance at the end of surgery. Penis is straightened and lengthened with urethral meatus positioned at the tip of the glans. 
Table 1 Surgical outcome 12 months after surgery

\begin{tabular}{|l|l|l|l|l|l|}
\hline No. of patient & Age (years) & $\begin{array}{l}\text { Preoperative } \\
\text { penis length in } \\
\text { erection }(\mathbf{c m})\end{array}$ & $\begin{array}{l}\text { Postoperative } \\
\text { penis length in } \\
\text { erection }(\mathbf{c m})\end{array}$ & $\begin{array}{l}\text { Residual penile } \\
\text { curvature } \\
\text { present }\end{array}$ & Sexually active \\
\hline 1 & 16 & 10.1 & 11.7 & - & - \\
\hline 2 & 17 & 10.3 & 12.4 & - & Yes \\
\hline 3 & 13 & 6.9 & 9.6 & Yes, mild & - \\
\hline 4 & 18 & 9.3 & 10.2 & - & Yes \\
\hline 5 & 15 & 9.6 & 11.5 & - & - \\
\hline 6 & 19 & 10.1 & 12.7 & - & Yes \\
\hline 7 & 18 & 9.8 & 11.9 & Yes, mild & Yes \\
\hline 8 & 14 & 7.6 & 9.8 & - & - \\
\hline 9 & 21 & 10.2 & 12.8 & - & Yes \\
\hline 10 & 17 & 8.9 & 10.1 & - & Yes \\
\hline 11 & 22 & 9.4 & 11.2 & - & Yes \\
\hline 12 & 16 & 7.8 & 9.3 & Yes, mild & Yes \\
\hline 13 & 18 & 9.5 & 11.7 & - & Yes \\
\hline
\end{tabular}

\section{Discussion}

Different surgical approaches have been applied in attempt to successfully repair epispadias, as one of the most severe penile anomaly. Regardless of the technique used, the goal of epispadias repair is reconstruction of functionally and aesthetically acceptable penis while attaining the maximum corporal length. Level of the defect and degree of the curvature impose the complexity of the surgical procedure. Although a large number of different surgical approaches were published, two major principles were widely accepted. The modified Cantwell-Ransley technique includes partial mobilization and tabularization of the urethra followed with rotation of the corporal bodies. ${ }^{2,3}$ Additional straightening was achieved by transversal incision of the tunica albuginea and longitudinal suturing of joined corporal bodies (cavernosocavernostomy). Surgical centers published their favorable results with modified Cantwell-Ransley procedure. ${ }^{4,5}$ Another widely accepted procedure was published by Mitchell and Bägli and includes penile disassembly with complete dissection of the urethral plate and separation of corporal bodies with its hemiglans. ${ }^{6}$ Complete dividing of the corporal bodies enables adequate medial rotation of the corpora with proper ventralization of the tubularized urethral plate and correction of the dorsal curvature. Many centers reported satisfactory results with complete disassembly procedure. ${ }^{7-9}$ Lengthening of the penis in disassembly procedure is achieved by excessive mobilization of freed corporal bodies. The urethra is brought to the glans if adequate length is present; otherwise, the resultant hypospadias can be repaired in a second stage. According to the published data, resultant hypospadias is fairly common and it was reported in 36 to $77 \%$ cases that underwent Mitchell-Bagli repair. ${ }^{9-12}$ Several modifications of standard disassembly procedure have been reported. ${ }^{13,14}$ In contrast to Mitchell-Bagli disas- sembly procedure, cornerstones of Perovic disassembly technique include complete detachment of corporal bodies from the glans and lifting of the neurovascular bundles. ${ }^{13}$ Marked dorsal curvature of the corporal bodies is corrected by incision on dorsal, concave ridge of the corpora, and grafting the defects. Perovic disassembly technique probably allows maximum of the possible lengthening because corpora are completely detached from urethra, glans, and neurovascular bundles. After that maneuver corporal bodies can be easily incised and grafted to obtain maximum length and complete straightening.

Complications after epispadias repair are not rare and include: fistula formation, urethral stricture, meatal stenosis, glans or wound dehiscence, residual curvature, skin necrosis, and severe ischemic injury with loss of the glans or corporeal body. ${ }^{15}$ Available reports usually present complications in short time follow-up and some of this reports are extended to 4 or 7 years postoperatively. ${ }^{5,9}$ However, there is no available data about delayed outcomes in patients who reached full sexual maturity. Some of the complications as residual dorsal curvature can become worsen after penile growth during puberty. Several authors noted that several complications after hypospadias repair may occur many years after initially successful outcome. ${ }^{16,17}$ It should be emphasized that followup of the patients who underwent reconstructive penile surgery should be extended until patients fulfill their sexual functionality.

Patients in our group underwent one to five procedures (median 2.2) during childhood for epispadias repair. All of them had severe scar formations that made disassembly procedure very difficult and challenging. Special care was taken to avoid iatrogenic vascular injury with possible disastrous consequences. Complete straightening and lengthening of the penis was imperative in our patients' expectations. Preoperatively, all patients in our group had 
severe dorsal curvature that would make sexual intercourse impossible or extremely awkward. Penile length is of major concern especially in pubertal males. Complete penile disassembly, with lifting of neurovascular bundles and transecting of short urethra, enabled full proximal mobilization of corporeal bodies. Finally, additional straightening and lengthening was easily achieved by incision of tunica albuginea on the dorsal side and grafting of the defects. Epispadiac penis is significantly shorter because of marked congenital deficiency of anterior corporal tissue. Therefore, maximum straightening and lengthening should be attempted to give the patients satisfactory result. Plication of ventral convex side of the corpora should be avoided as it inevitably leads to additional shortening of the penis. Potentially, the best results can be obtained by maximal proximal mobilization of the corpora and grafting of the shorter side of the corporal bodies. Postoperatively, usage of vacuum device was advised to our patients to attain maximal penile length and to prevent retraction of the graft and scar tissue.

Regardless of the technique used, resultant hypospadias repair can be a difficult surgical task with high complication rate. Simultaneous use of hairless local skin flap and buccal mucosa free graft enables one-stage urethral reconstruction in these patients. Dorsal half of newly created urethra was formed by buccal mucosa graft and ventral half by longitudinal skin flap, therefore minimizing possibility for urethral strictures. Wide pedicle of the skin flap was fixed over the lateral suture lines to prevent fistula formation.

\section{Conclusion}

Redo epispadias repair after failed surgery in childhood is very challenging and demanding procedure. Lack of available healthy tissue and presence of scar formation impose careful surgical approach. Complete penile disassembly enables full correction of all deformities, primarily marked dorsal curvature, and short penile shaft. Radical approach in redo epispadias repair is necessary to achieve functionally and aesthetically satisfactory result, but it should be performed only by experienced and skilful team in highly specialized surgical centers.

\section{Acknowledgment}

This article is supported by the Ministry of Science, Republic of Serbia; Project number 175048.

\section{Conflict of Interest}

None

\section{References}

1 Gearhart JP, Mathews RI. Exstrophy-epispadias complex. In: Wein AJ, ed. Campbell-Walsh Urology. 10th ed. Philadelphia, PA: Saunders Elsevier; 2011

2 Cantwell FV. Operative treatment of epispadias by transplantation of the urethra. Ann Surg 1895;22(6):689-694

3 Ransley PG, Duffy PG, Wollin M. Bladder exstrophy closure and epispadias repair. In: Spitz L, Nixon HH, eds. Rob and Smith's Operative Surgery: Pediatric Surgery. Boston, MA: Butterworths; 1989:620-622

4 Surer I, Baker LA, Jeffs RD, Gearhart JP. The modified CantwellRansley repair for exstrophy and epispadias: 10-year experience. J Urol 2000;164(3 Pt 2):1040-1042, discussion 1042-1043

5 Baird AD, Gearhart JP, Mathews RI. Applications of the modified Cantwell-Ransley epispadias repair in the exstrophy-epispadias complex. J Pediatr Urol 2005;1(5):331-336

6 Mitchell ME, Bägli DJ. Complete penile disassembly for epispadias repair: the Mitchell technique. J Urol 1996;155(1):300-304

7 Zaontz MR, Steckler RE, Shortliffe LM, Kogan BA, Baskin L, Tekgul S. Multicenter experience with the Mitchell technique for epispadias repair. J Urol 1998;160(1):172-176

8 Grady RW, Mitchell ME. Management of epispadias. Urol Clin North Am 2002;29(2):349-360, vi

9 Kibar Y, Roth C, Frimberger D, Kropp BP. Long-term results of penile disassembly technique for correction of epispadias. Urology 2009;73(3):510-514

10 Hafez AT, El-Sherbiny MT, Shorrab AA, El-Mowafi H. Complete primary repair of bladder exstrophy in children presenting late and those with failed initial closure: single center experience. J Urol 2005;174(4 Pt 2):1549-1552, discussion 1552

11 Hafez AT, El-Sherbiny MT. Complete repair of bladder exstrophy: management of resultant hypospadias. J Urol 2005;173(3): 958-961

12 Hafez AT, Helmy T. Complete penile disassembly for epispadias repair in postpubertal patients. Urology 2011;78(6):1407-1410

13 Perovic SV, Vukadinovic V, Djordjevic ML, Djakovic NG. Penile disassembly technique for epispadias repair: variants of technique. J Urol 1999;162(3 Pt 2):1181-1184

14 El-Sherbiny MT, Hafez AT. Complete repair of bladder exstrophy in boys: can hypospadias be avoided? Eur Urol 2005;47(5):691-694

15 Husmann DA, Gearhart JP. Loss of the penile glans and/or corpora following primary repair of bladder exstrophy using the complete penile disassembly technique. J Urol 2004;172(4 Pt 2):1696-1700, discussion 1700-1701

16 Tang SH, Hammer CC, Doumanian L, Santucci RA. Adult urethral stricture disease after childhood hypospadias repair. In: Djordjevic ML, ed. Penile Reconstructive Surgery. Advances in Urology. New York, NY: Hindawi Publishing Corporation; 2008:1-4

17 Barbagli G, De Angelis M, Palminteri E, Lazzeri M. Failed hypospadias repair presenting in adults. Eur Urol 2006;49(5):887-894, discussion 895 